\title{
REGULARITY OF MINIMIZERS FOR NONCONVEX VECTORIAL INTEGRALS WITH $p-q$ GROWTH VIA RELAXATION METHODS
}

IRENE BENEDETTI AND ELVIRA MASCOLO

Received 10 January 2003

Local Lipschitz continuity of local minimizers of vectorial integrals $\int_{\Omega} f(x, D u) d x$ is proved when $f$ satisfies $p-q$ growth condition and $\xi \mapsto f(x, \xi)$ is not convex. The uniform convexity and the radial structure condition with respect to the last variable are assumed only at infinity. In the proof, we use semicontinuity and relaxation results for functionals with nonstandard growth.

\section{Introduction}

In this paper, we study the regularity properties of the local minimizers of nonhomogeneous integral functionals of the form

$$
I(u, \Omega)=\int_{\Omega} f(x, D u) d x
$$

where $\Omega \subset \mathbb{R}^{n}$ is a bounded open set and $D u$ denotes the gradient matrix of a vectorvalued function $u: \Omega \rightarrow \mathbb{R}^{m}, n, m>1$.

We say that a function $u \in W_{\text {loc }}^{1,1}\left(\Omega, \mathbb{R}^{m}\right)$ is a local minimizer of $I$ if $f(x, D u) \in L_{\text {loc }}^{1}(\Omega)$ and

$$
I(u, \operatorname{supp} \varphi) \leq I(u+\varphi, \operatorname{supp} \varphi),
$$

for any $\varphi \in W^{1,1}\left(\Omega, \mathbb{R}^{m}\right)$ with $\operatorname{supp} \varphi \Subset \Omega$.

The main features of our functional $I$ is the fact that the density energy $f=f(x, \xi)$ is not convex with respect to $\xi \in \mathbb{R}^{n m}$ and satisfies the so-called $p$ - $q$ growth condition, that is, there exist $1<p<q$ and $c_{0}, c_{1}, L>0$, such that

$$
c_{1}|\xi|^{p}-c_{0} \leq f(x, \xi) \leq L\left(1+|\xi|^{q}\right) .
$$

The regularity of minimizers in the $p-q$ growth condition (1.3) has been extensively studied in the last years, starting from some papers of Marcellini, see for example $[11,12]$. 
The study is motivated by several problems in different contexts of mathematical physics as, for example, in nonlinear elasticity and homogenization.

Most of the regularity results in the vectorial case $m>1$ are obtained under the assumptions

$$
f(x, \xi)=g(x,|\xi|) \quad x \in \Omega, \xi \in \mathbb{R}^{n m},
$$

with $g: \Omega \times[0, \infty[\rightarrow[0, \infty[$ and $f$ convex with respect to $\xi$. For example, in a recent paper, Cupini et al. [2] proved that if (1.3), with $\xi \in \mathbb{R}^{n m}$, and (1.4), with $\xi \in \mathbb{R}^{n m} \backslash$ $B_{R}(0)$, hold and, in addition, $f$ is $p$-uniformly convex at infinity, the local minimizers of $I$ are Lipschitz continuous when $1<p \leq q<p(n+1) / n$. If $q>p(n+1) / n$, there are some counterexamples to the regularity. See also Esposito et al. [6] and Mascolo and Migliorini [13] for related results.

The regularity of minimizers of nonconvex functionals is achieved, usually, via relaxation methods. For instance, Fonseca et al. [7], in scalar case $m=1$ and in standard growth $p=q$, observed that, by the known relaxation theorems, local minimizers of nonconvex functionals are also minimizers of

$$
I^{* *}(u, \Omega)=\int_{\Omega} f^{* *}(x, D u) d x
$$

where $f^{* *}$ is the convex envelope of $f$ with respect to $\xi$. Thus they can reduce to study the regularity of minimizers of convex functionals; see also Cupini and Migliorini [3].

However, in the vectorial case, the relaxation methods are not so well clarified when dealing with $p$ - $q$ growth condition.

The lower semicontinuity with respect to the weak topology $W^{1, p}\left(\Omega, \mathbb{R}^{m}\right)$ of quasiconvex functional, with integrand satisfying (1.3), has been studied by Marcellini [12] for $f=f(x, \xi)$ and $q<p(n+1) / n$ and by Fonseca and Malỳ [8] when $f=f(\xi)$ and $q<p n /(n-1)$. Further in [8], a representation of the relaxed functional is given when $Q f(\xi)=f^{* *}(\xi)$, where $Q f(\xi)$ denotes the quasiconvex envelope of $f$.

Here we consider nonhomogeneous density energies $f=f(x, \xi)$, which are Caratheodory functions satisfying a local continuity condition with respect to $x$ (see assumption (A2)). First, we prove a lower semicontinuity theorem when $f$ is quasiconvex and $q<$ $p n /(n-1)$ (see Theorem 2.2). Moreover, when

$$
Q f(x, \xi)=f^{* *}(x, \xi),
$$

as in [8], we are able to prove that (see Theorem 2.6) for all $U \Subset \Omega$ and $u \in W^{1, p}\left(\Omega, \mathbb{R}^{m}\right)$,

$$
\mathscr{F}^{p} p, q(u, U)=I^{* *}(u, U),
$$

where

$$
\begin{aligned}
\mathscr{F}^{p}, q(u, \Omega)=\inf _{u_{k}}\left\{\liminf _{k \rightarrow \infty} \int_{\Omega} f\left(x, D u_{k}\right) d x,\right. \\
\left.\left(u_{k}\right) \in W^{1, q}\left(\Omega, \mathbb{R}^{m}\right), u_{k} \rightarrow u \text { in } W^{1, p}\left(\Omega, \mathbb{R}^{m}\right)\right\} .
\end{aligned}
$$

Our main regularity result follows by equality (1.7). 
Let $f$ be not convex in $\xi$, p-uniformly convex at infinity, and satisfy the conditions (1.4) and (1.6). Then, in Theorem 3.2, we prove that all local minimizers of $I$ are local Lipschitz continuous. We give a sketch of the proof.

Let $u$ be a local minimizer of $I(u)$ and $U \Subset \Omega$. Consider

$$
I(u, U)=\inf \left\{I(v, U): v \in W_{0}^{1, p}\left(U, \mathbb{R}^{m}\right)+u\right\}
$$

and the convex problem

$$
\inf \left\{I^{* *}(v, U): v \in W_{0}^{1, p}\left(U, \mathbb{R}^{m}\right)+u\right\} .
$$

For classical results, (1.10) has at least one solution $\bar{u}$ and [2, Theorem 1.1] implies that $\bar{u} \in W_{\text {loc }}^{1, \infty}\left(U, \mathbb{R}^{m}\right)$. We prove that

$$
I(u, U)=I^{* *}(\bar{u}, U)=I^{* *}(u, U) .
$$

The proof of the last equalities is based on the representation formula (1.7), on a method introduced by De Giorgi [5], and on the related ideas contained in Fusco [9] and in Marcellini [12]. Then, since (1.11) implies that $u$ is a solution of the convex problem (1.10), we get $u \in W_{\text {loc }}^{1, \infty}\left(U, \mathbb{R}^{m}\right)$.

Moreover, we exhibit a class of density energies for which (1.6) is satisfied.

Finally, we study the relationship between (1.6) and the Lavrentiev phenomenon. In particular, we show that under (1.4) and (1.6), we do not have the occurrence of the phenomenon.

In conclusion, we observe that, in the scalar case, the regularity of minimizers for nonconvex functionals is often the first step to prove the existence, see Mascolo and Schianchi [14] and Fonseca et al. [7], then it would be interesting to complete these researches in that direction.

This paper is organized as follows. In Section 2, we give the proof of the semicontinuity theorem and the characterization of $\mathscr{F}^{p, q}$ and Section 3 is devoted to the study of the regularity of local minimizers.

\section{Semicontinuity and relaxation}

Let $\Omega \subset \mathbb{R}^{n}$ be an open bounded set and let $f: \Omega \times \mathbb{R}^{n m} \rightarrow \mathbb{R}$ be a nonnegative Caratheodory function satisfying the following assumptions:

(A1) there exist $q>1$ and $L>0$ such that

$$
0 \leq f(x, \xi) \leq L\left(1+|\xi|^{q}\right)
$$

for almost everywhere $x \in \Omega$ and for every $\xi \in \mathbb{R}^{n m}$;

(A2) there exists a modulus of continuity $\lambda(t)$ (i.e., $\lambda(t)$ is a nonnegative increasing function that goes to zero as $t \rightarrow 0^{+}$) such that for every compact subset $\Omega_{0} \subset \Omega$, there exists $x_{0} \in \Omega_{0}$ such that

$$
\left|f\left(x_{0}, \xi\right)-f(x, \xi)\right| \leq \lambda\left(\left|x-x_{0}\right|\right)(1+f(x, \xi)),
$$

for all $x \in \Omega_{0}$ and $\xi \in \mathbb{R}^{n m}$. 
Remark 2.1. It is easy to check that (2.2) implies that there exists $\delta>0$, with $\lambda(\delta)<1$, such that when $\Omega_{0} \subset \Omega$ is a compact set with $\operatorname{diam}\left(\Omega_{0}\right)<\delta$, there exists a modulus of continuity $\mu(t)$ in $[0, \delta[$ such that

$$
\left|f\left(x_{0}, \xi\right)-f(x, \xi)\right| \leq \mu\left(\left|x-x_{0}\right|\right)\left(1+f\left(x_{0}, \xi\right)\right),
$$

for all $x \in \Omega_{0}$ and for all $\xi \in \mathbb{R}^{n m}$.

In fact, fixing $\xi \in \mathbb{R}^{n m}$ for all $x \in \Omega$ such that $f\left(x_{0}, \xi\right) \geq f(x, \xi)$, the condition (2.2) implies (2.3) with $\mu=\lambda$.

Let $x \in \Omega_{0}$ be such that $f(x, \xi)>f\left(x_{0}, \xi\right)$, then (2.2) can be written as

$$
f\left(x_{0}, \xi\right) \geq f(x, \xi)\left[1-\lambda\left(\left|x-x_{0}\right|\right)\right]-\lambda\left(\left|x-x_{0}\right|\right)
$$

Thus if $\delta$ is such that $\lambda(\delta)<1$, then for all $\Omega_{0} \Subset \Omega$ with $\operatorname{diam}\left(\Omega_{0}\right)<\delta$, (2.3) holds with $\mu(t)=\lambda(t) /(1-\lambda(t))$.

We say that a function $f(x, \xi)$ is quasiconvex with respect to $\xi$ in the Morrey's sense if for every $x \in \Omega$ and $\xi \in \mathbb{R}^{n m}$,

$$
\int_{\Omega} f(x, \xi+D \varphi(y)) d y \geq|\Omega| f(x, \xi) \quad \forall \varphi \in W_{0}^{1, \infty}\left(\Omega, \mathbb{R}^{m}\right) .
$$

First, we prove the following lower semicontinuity theorem in Sobolev spaces below the growth exponent for the density energy.

Theorem 2.2. Let $f$ be a quasiconvex function satisfying (A1) and (A2) and let $1<p<q<$ $n /(n-1) p$. Then

$$
\liminf _{k \rightarrow \infty} \int_{\Omega} f\left(x, D u_{k}\right) d x \geq \int_{\Omega} f(x, D u) d x
$$

for all $u \in W^{1, p}\left(\Omega, \mathbb{R}^{m}\right)$, with $f(x, D u) \in L^{1}(\Omega)$, and for all $u_{k} \in W^{1, q}\left(\Omega, \mathbb{R}^{m}\right)$ such that $u_{k}$ converges to $u$ in the weak topology of $W^{1, p}\left(\Omega, \mathbb{R}^{m}\right)$.

Proof. Let $u \in W^{1, p}\left(\Omega, \mathbb{R}^{m}\right)$ and $u_{k} \rightarrow u$ in $W^{1, p}$.

Without loss of generality we may assume that

$$
\liminf _{k \rightarrow \infty} \int_{\Omega} f\left(x, D u_{k}\right) d x<\infty
$$

Let $\Omega_{0}$ be an open set compactly contained in $\Omega$. For every integer $\nu$ such that $\lambda(1 / \nu)<1$, we consider a subdivision of $\Omega_{0}$ in open sets $\Omega_{i}$ such that $\Omega_{i} \cap \Omega_{j}=\varnothing$ for $i \neq j, \sum_{i}\left|\Omega_{i}\right|=$ $\left|\Omega_{0}\right|$, and $\operatorname{diam} \Omega_{i}<1 / \nu$. By (A2) and Remark 2.1, there exists $x_{i} \in \overline{\Omega_{i}}$ for which

$$
\begin{aligned}
& \left|f\left(x_{i}, \xi\right)-f(x, \xi)\right| \leq \lambda\left(\frac{1}{\nu}\right)(1+f(x, \xi)), \\
& \left|f\left(x_{i}, \xi\right)-f(x, \xi)\right| \leq \mu\left(\frac{1}{\nu}\right)\left(1+f\left(x_{i}, \xi\right)\right),
\end{aligned}
$$


for all $x \in \overline{\Omega_{i}}$ and $\xi \in \mathbb{R}^{n m}$. Then by using the second inequality of (2.8)

$$
\begin{aligned}
\int_{\Omega_{0}} f(x, D u) d x & \leq \sum_{i} \int_{\Omega_{i}} f(x, D u) d x \\
& \leq \sum_{i} \int_{\Omega_{i}} f\left(x_{i}, D u\right) d x+\mu\left(\frac{1}{v}\right) \sum_{i} \int_{\Omega_{i}}\left[1+f\left(x_{i}, D u\right)\right] d x .
\end{aligned}
$$

We estimate the first integral in the right-hand side. Since $q<p n /(n-1)$, by the lower semicontinuity result for functionals with homogeneous density energy contained in $[8$, Theorem 4.1], we have

$$
\int_{\Omega_{i}} f\left(x_{i}, D u\right) d x \leq \liminf _{k \rightarrow \infty} \int_{\Omega_{i}} f\left(x_{i}, D u_{k}\right) d x .
$$

The first inequality of (2.8) implies that

$$
\begin{aligned}
\sum_{i} \liminf _{k \rightarrow \infty} \int_{\Omega_{i}} f\left(x_{i}, D u_{k}\right) d x & \leq \sum_{i} \liminf _{k \rightarrow \infty} \int_{\Omega_{i}}\left\{\left[1+\lambda\left(\frac{1}{v}\right)\right] f\left(x, D u_{k}\right)+\lambda\left(\frac{1}{v}\right)\right\} d x \\
& \leq\left[1+\lambda\left(\frac{1}{v}\right)\right] \liminf _{k \rightarrow \infty} \int_{\Omega_{0}} f\left(x, D u_{k}\right) d x+\lambda\left(\frac{1}{v}\right)\left|\Omega_{0}\right|
\end{aligned}
$$

By (2.7) and (2.10), there exists $M>0$ such that

$$
\sum_{i} \int_{\Omega_{i}} f\left(x_{i}, D u\right) d x<M
$$

and consequently we obtain

$$
\begin{aligned}
\int_{\Omega_{0}} f(x, D u) d x \\
\quad \leq\left[1+\lambda\left(\frac{1}{v}\right)\right] \liminf _{k \rightarrow \infty} \int_{\Omega_{0}} f\left(x, D u_{k}\right) d x+\lambda\left(\frac{1}{v}\right)\left|\Omega_{0}\right|+\mu\left(\frac{1}{v}\right)\left[M+\left|\Omega_{0}\right|\right] .
\end{aligned}
$$

Thus we go to the limit as $\nu \rightarrow \infty$ and then we get the result as $\Omega_{0} \uparrow \Omega$.

Suppose that $f$ satisfies (A1) and (A2) and let $Q f(x, \xi)$ be the quasiconvex envelope of $f$ with respect to the second variable, that is,

$$
Q f(x, \xi)=\sup \{g \leq f: g \text { quasiconvex with respect to } \xi\} \text {. }
$$

By the results contained in Dacorogna [4] and in Giusti [10], we have that (A1) implies

$$
Q f(x, \xi)=\inf \left\{\frac{1}{|\Omega|} \int_{\Omega} f(x, \xi+D \varphi(y)) d y \forall \varphi \in W_{0}^{1, q}\right\} .
$$

By the definition of $Q f$, we have that

$$
0 \leq Q f(x, \xi) \leq L\left(1+|\xi|^{q}\right)
$$

Now we show that when $f$ satisfies (A2), $Q f$ satisfies the same property. 
Lemma 2.3. Let $f: \Omega \times \mathbb{R}^{n m} \rightarrow \mathbb{R}$ satisfy (A1) and (A2); then there exists $\delta>0$ such that for every compact subset $\Omega_{0}$ of $\Omega$ with $\operatorname{diam}\left(\Omega_{0}\right)<\delta$, there exists $x_{0} \in \Omega_{0}$ such that

$$
\left|Q f\left(x_{0}, \xi\right)-Q f(x, \xi)\right| \leq \mu\left(\left|x-x_{0}\right|\right)(1+Q f(x, \xi)),
$$

for all $x \in \Omega_{0}$ and for all $\xi \in \mathbb{R}^{n m}$, where $\mu(t)=\lambda(t) /(1-\lambda(t))$, for $0<t<\delta$.

Proof. By the characterization of the quasiconvex envelope (2.15), we have that, for $x \in$ $\Omega$, there exists a sequence $\left(\varphi_{x j}\right) \in W_{0}^{1, q}\left(\Omega, \mathbb{R}^{m}\right)$ such that

$$
Q f(x, \xi) \leq \frac{1}{|\Omega|} \int_{\Omega} f\left(x, \xi+D \varphi_{x j}(y)\right) d y \leq Q f(x, \xi)+\frac{1}{j} .
$$

Let $\Omega_{0} \Subset \Omega$ be a compact subset and let $x_{0} \in \Omega_{0}$ satisfy (2.2):

$$
\begin{aligned}
& Q f\left(x_{0}, \xi\right)-Q f(x, \xi) \\
& \quad \leq \frac{1}{|\Omega|} \int_{\Omega} f\left(x_{0}, \xi+D \varphi_{x j}(y)\right) d y-\frac{1}{|\Omega|} \int_{\Omega} f\left(x, \xi+D \varphi_{x j}(y)\right) d y+\frac{1}{j} \\
& \quad \leq \lambda\left(\left|x-x_{0}\right|\right)(1+Q f(x, \xi))+\frac{1}{j}\left(\lambda\left(\left|x-x_{0}\right|\right)+1\right) ;
\end{aligned}
$$

as $j \rightarrow \infty$, we obtain

$$
Q f\left(x_{0}, \xi\right)-Q f(x, \xi) \leq \lambda\left(\left|x-x_{0}\right|\right)(1+Q f(x, \xi)) .
$$

Now let $\left(\varphi_{x_{0} j}\right) \in W_{0}^{1, q}\left(\Omega, \mathbb{R}^{m}\right)$ satisfy (2.18) with $x_{0}$ in place of $x$. With the same previous arguments, by applying (2.3) instead of (2.2), for $\Omega_{0}$ with $\operatorname{diam}\left(\Omega_{0}\right)<\delta$ (with $\delta$ chosen such that $\lambda(\delta)<1)$, we get

$$
Q f(x, \xi)-Q f\left(x_{0}, \xi\right) \leq \mu\left(\left|x-x_{0}\right|\right)\left(1+Q f\left(x_{0}, \xi\right)\right),
$$

so (2.20) and (2.21) imply (2.17).

Remark 2.4. Let $f(x, \xi)=a(x) g(\xi)$, where $g: \mathbb{R}^{n m} \rightarrow \mathbb{R}$ has $q$-growth:

$$
0<g(\xi)<L\left(1+|\xi|^{q}\right)
$$

where $L>0$ is a constant and $a \in C^{0, \alpha}(\Omega)$. In this case, both $f$ and $Q f$ satisfy (A1) and (A2); in fact $Q f(x, \xi)=a(x) Q g(\xi)$.

We introduce the functional $\mathscr{F} p, q$ :

$$
\begin{aligned}
\mathscr{F}^{p}, q(u)=\inf _{u_{k}}\left\{\liminf _{k \rightarrow \infty} \int_{\Omega} f\left(x, D u_{k}\right) d x,\right. \\
\left.\left(u_{k}\right) \in W^{1, q}\left(\Omega, \mathbb{R}^{m}\right), u_{k} \rightarrow u \text { in } W^{1, p}\left(\Omega, \mathbb{R}^{m}\right)\right\} .
\end{aligned}
$$

The following theorem holds. 
Theorem 2.5. Let $1<p<q<p n /(n-1)$, let $f$ satisfy (A1) and (A2), and $Q f(x, \xi) \in$ $L^{1}(\Omega)$. Then for $u \in W^{1, p}\left(\Omega, \mathbb{R}^{m}\right)$,

$$
\mathscr{F}^{p, q} \geq \int_{\Omega} Q f(x, D u) d x .
$$

When $u \in W^{1, q}\left(\Omega, \mathbb{R}^{m}\right)$, the equality in (2.24) holds.

Proof. It is not difficult to check that the arguments in the proof of Theorem 2.2 can be applied to the quasiconvex functional $\int_{\Omega} Q f(x, D u) d x$ by choosing a decomposition of $\Omega_{0}$ in open set $\Omega_{i}$, with $\operatorname{diam}\left(\Omega_{i}\right)<1 / \nu$, where $\nu$ is sufficiently big, so that $\Omega_{i}$ satisfies the condition of Lemma 2.3. Then if $\left(u_{k}\right) \in W^{1, q}\left(\Omega, \mathbb{R}^{m}\right)$ and $u_{k} \rightarrow u$ in $W^{1, p}\left(\Omega, \mathbb{R}^{m}\right)$, we obtain

$$
\liminf _{k \rightarrow \infty} \int_{\Omega} f\left(x, D u_{k}\right) d x \geq \liminf _{k \rightarrow \infty} \int_{\Omega} Q f\left(x, D u_{k}\right) d x \geq \int_{\Omega} Q f(x, D u) d x ;
$$

taking the infimum over all such sequences, (2.24) follows.

When $u \in W^{1, q}\left(\Omega, \mathbb{R}^{m}\right)$, by the standard relaxation results, there exists a sequence $\left(w_{j}\right) \in W^{1, q}\left(\Omega, \mathbb{R}^{m}\right)$ such that $w_{j} \rightarrow u$ in $W^{1, q}\left(\Omega, \mathbb{R}^{m}\right)$ and

$$
\liminf _{j \rightarrow \infty} \int_{\Omega} f\left(x, D u_{j}\right) d x=\int_{\Omega} Q f(x, D u) d x
$$

which easily implies that (2.24) is an equality.

Assume now a $p$-coercivity condition on $f$.

$\left(\mathrm{A} 1^{\prime}\right)$ There exist $c_{0}, c_{1}, L>0$ such that

$$
c_{1}|\xi|^{p}-c_{0} \leq f(x, \xi) \leq L\left(1+|\xi|^{q}\right) .
$$

In the following, $f^{* *}(x, \xi)$ denotes the lower convex envelope of $f$ with respect to the second variable.

We are able now to give a characterization of $\mathscr{F}_{p} p, q$.

Theorem 2.6. Let $f$ satisfy $\left(A 1^{\prime}\right)$ and $(A 2)$ and let $1<p<q<p(n+1) / n$. Assume that $Q f(x, \xi)$ is a convex function with respect to $\xi$, that is, $Q f(x, \xi)=f^{* *}(x, \xi)$. Then, for all $u \in W^{1, p}\left(\Omega, \mathbb{R}^{m}\right)$ such that $Q f(x, D u) \in L_{\mathrm{loc}}^{1}(\Omega)$,

$$
\left.\mathscr{F}^{p} p, q, U\right)=\int_{U} Q f(x, D u) d x
$$

for all $U \Subset \Omega$, open set.

Proof. Consider a smooth kernel $\varphi \geq 0$ in $\mathbb{R}^{m}$ with support in $\overline{B(0,1)}, \int_{\mathbb{R}^{n}} \varphi(x) d x=1$, and $\varphi_{j}(x)=j^{n} \varphi(j x)$. For each $j \in \mathbb{N}$, consider $\varphi_{j} * u \in W^{1, q}\left(U, \mathbb{R}^{m}\right)$. Again for the standard relaxation results, we can select a sequence $v_{j k} \in W^{1, q}\left(U, \mathbb{R}^{m}\right)$ such that $\lim _{k \rightarrow \infty} v_{j k}=$ $\varphi_{j} * u$ weakly in $W^{1, q}\left(U, \mathbb{R}^{m}\right)$ and

$$
\lim _{k \rightarrow \infty} \int_{U} f\left(x, D v_{j k}\right) d x=\int_{U} Q f\left(x, D\left(\varphi_{j} * u\right)\right) d x .
$$


34 Regularity of minimizers for nonconvex integrals

We may extract a diagonal subsequence $u_{j}=v_{j k(j)}$ such that $u_{j} \rightarrow u$ in $W^{1, p}\left(U, \mathbb{R}^{m}\right)$ and

$$
\left|\int_{U} f\left(x, D u_{j}\right) d x-\int_{U} Q f\left(x, D\left(\varphi_{j} * u\right)\right) d x\right| \leq \frac{1}{j} .
$$

For every positive $\nu \in \mathbb{N}$ with $\nu \geq \bar{v}=\inf \{\nu: \lambda(1 / \nu)<1\}$, we consider a subdivision of $U$ in the open sets $U_{i}$ such that $\sum_{i}\left|U_{i}\right|=|U|, U_{i} \cap U_{j}=\varnothing$, for $i \neq j$ and $\operatorname{diam}\left(U_{i}\right)<1 / \nu \forall i$. We have, from (2.21),

$$
\begin{aligned}
\liminf _{j \rightarrow \infty} & \int_{U} Q f\left(x, D\left(\varphi_{j} * u\right)\right) d x \\
= & \liminf _{j \rightarrow \infty} \sum_{i} \int_{U_{i}} Q f\left(x, D\left(\varphi_{j} * u\right)\right) d x \\
\leq & \liminf _{j \rightarrow \infty} \sum_{i} \mu\left(\frac{1}{v}\right) \int_{U_{i}}\left[1+Q f\left(x_{i}, D\left(\varphi_{j} * u\right)\right)\right] d x \\
& +\liminf _{j \rightarrow \infty} \sum_{i} \int_{U_{i}} Q f\left(x_{i}, D\left(\varphi_{j} * u\right)\right) \\
= & \mu\left(\frac{1}{v}\right)|U|+\left(1+\mu\left(\frac{1}{v}\right)\right) \liminf _{j \rightarrow \infty} \sum_{i} \int_{U_{i}} Q f\left(x_{i}, D\left(\varphi_{j} * u\right)\right) d x
\end{aligned}
$$

Since $Q f$ is convex and the measure $\mu_{x}^{j}$, given by

$$
\left\langle\mu_{x}^{j}, v\right\rangle:=\int_{U} \varphi_{j}(x-y) v(y) d y
$$

is a probability measure, using Jensen inequality, we have, for fixed $i$,

$$
\int_{U_{i}} Q f\left(x_{i}, D\left(\varphi_{j} * u\right)\right)=\int_{U_{i}} Q f\left(x_{i},\left\langle\mu_{x}^{j}, D u\right\rangle\right) d x \leq \int_{U_{i}}\left\langle\mu_{x}^{j}, Q f\left(x_{i}, D u\right)\right\rangle d x .
$$

Since $Q f\left(x_{i}, D u\right) \in L^{1}(\Omega)$, by a known result, we get

$$
\int_{U_{i}}\left\langle\mu_{x}^{j}, Q f\left(x_{i}, D u\right)\right\rangle d x \leq \int_{U_{i}} Q f\left(x_{i}, D u\right) d x,
$$

then, by (2.20),

$$
\begin{aligned}
\liminf _{j \rightarrow \infty} & \int_{U} Q f\left(x, D\left(\varphi_{j} * u\right)\right) \\
\leq & \mu\left(\frac{1}{v}\right)|U|+\left(1+\mu\left(\frac{1}{v}\right)\right) \sum_{i} \int_{U_{i}} Q f\left(x_{i}, D u\right) d x \\
\leq & \mu\left(\frac{1}{v}\right)|U|+\left(1+\mu\left(\frac{1}{v}\right)\right) \lambda\left(\frac{1}{v}\right) \sum_{i} \int_{U_{i}}(1+Q f(x, D u)) d x \\
& +\left(1+\mu\left(\frac{1}{v}\right)\right) \int_{U} Q f(x, D u) d x .
\end{aligned}
$$


As $v$ goes to $\infty$, since $\int_{U} Q f(x, D u) d x<\infty$, we get

$$
\liminf _{j \rightarrow \infty} \int_{U} Q f\left(x, D\left(\varphi_{j} * u\right)\right) d x \leq \int_{U} Q f(x, D u) d x .
$$

Then, by (2.30),

$$
\begin{aligned}
\mathscr{F}^{p} p, q(u, U) & \leq \liminf _{j \rightarrow \infty} \int_{U} f\left(x, D u_{j}\right) d x \\
& =\liminf _{j \rightarrow \infty} \int_{U} Q f\left(x, D\left(\varphi_{j} * u\right)\right) d x \\
& \leq \int_{U} Q f(x, D u) d x,
\end{aligned}
$$

so by (2.24), (2.28) holds.

Remark 2.7. Under the hypotheses of Theorem 2.6, consider the following functional:

$$
\begin{array}{r}
\mathscr{F}(u, \Omega)=\inf _{u_{k}}\left\{\liminf _{k \rightarrow \infty} \int_{\Omega} f\left(x, D u_{k}\right) d x,\left(u_{k}\right) \in W^{1, p}\left(\Omega, \mathbb{R}^{m}\right),\right. \\
\left.u_{k} \rightarrow u \text { in } W^{1, p}\left(\Omega, \mathbb{R}^{m}\right), f\left(x, D u_{k}\right) \in L^{1}(\Omega)\right\} .
\end{array}
$$

For all $U \Subset \Omega$, we have

$$
\mathscr{F}^{p, q}(u, U)=\mathscr{F}(u, U) .
$$

Indeed for all $u_{k} \in W^{1, p}\left(U, \mathbb{R}^{m}\right)$ with $f\left(x, D u_{k}\right) \in L^{1}(U)$ and $u_{k} \rightarrow u$ in $W^{1, p}\left(\Omega, \mathbb{R}^{m}\right)$, we have

$$
\int_{\Omega} f^{* *}(x, D u) d x \leq \liminf _{k \rightarrow \infty} \int_{\Omega} f^{* *}\left(x, D u_{k}\right) d x \leq \liminf _{k \rightarrow \infty} \int_{\Omega} f\left(x, D u_{k}\right) d x .
$$

Therefore,

$$
\mathscr{F}(u, U) \leq \mathscr{F}^{p}, q(u, U)=\int_{U} f^{* *}(x, D u) \leq \mathscr{F}(u, U) .
$$

Now we exhibit a class of integrands $f: \Omega \times \mathbb{R}^{n m} \rightarrow \mathbb{R}$ such that $Q f(x, A)=f^{* *}(x, A)$, for every $A \in \mathbb{R}^{n m}$.

We say that $f: \Omega \times \mathbb{R}^{n m} \rightarrow \mathbb{R}$ is rank-one convex if, for every $x \in \Omega$,

$$
f(x, \lambda A+(1-\lambda) B) \leq \lambda f(x, A)+(1-\lambda) f(x, B),
$$

for all $\lambda \in[0,1]$ and $A, B \in \mathbb{R}^{n m}$ with $\operatorname{rank}(A-B) \leq 1$.

For $f: \Omega \times \mathbb{R}^{n m} \rightarrow \mathbb{R}$, we define the rank-one convex envelope as

$$
R f(x, A)=\sup \{g \leq f: g \text { rank-one convex with respect to } A\} .
$$

We prove the following result. 
Proposition 2.8. Let $\Omega$ be a bounded open set of $\mathbb{R}^{n}$ and let $f: \Omega \times \mathbb{R}^{n m} \rightarrow \mathbb{R}$. Assume that there exists a function $g: \Omega \times \mathbb{R}_{+} \rightarrow \mathbb{R}$ such that

$$
\begin{gathered}
f(x, A)=g(x,|A|), \\
g(x, t)=g(x,-t),
\end{gathered}
$$

for every $x \in \Omega$ and for every $t>0$.

Moreover, assume that there exists a measurable, nonnegative function $\alpha: \Omega \rightarrow \mathbb{R}_{+}$such that

$$
g^{* *}(x, t)=g(x, \alpha(x))
$$

for all $t$ with $|t|<\alpha(x)$ and

$$
g^{* *}(x, t)=g(x, t)
$$

for all $t \geq \alpha(x)$.

Then,

$$
g^{* *}(x,|A|)=f^{* *}(x,|A|)=Q f(x,|A|)=R f(x,|A|) .
$$

Proof. We use the same techniques contained in Dacorogna [4]. First, we prove that $g^{* *}(x,|A|) \geq f^{* *}(x,|A|)$.

Let $x \in \Omega$ and let $\varepsilon$ be fixed, then by the characterization of the convex envelope, there exist $\lambda \in[0,1]$ and $b, c \in \mathbb{R}_{+}$such that

$$
\begin{aligned}
\varepsilon+g^{* *}(x,|A|) & \geq \lambda g(x, b)+(1-\lambda) g(x, c), \\
|A| & =\lambda b+(1-\lambda) c .
\end{aligned}
$$

Choosing $B=b A /|A|$ and $C=c A /|A|$, we get

$$
\varepsilon+g^{* *}(x,|A|) \geq \lambda f(x, B)+(1-\lambda) f(x, C) \geq f^{* *}(x,|A|) .
$$

From the arbitrariness of $\varepsilon$, we have the claimed result. To prove the reverse inequality, we first show that $(2.45)$ implies that $g^{* *}(x, t)$ is not decreasing with respect to $t$.

Fixing $x \in \Omega$, we have

$$
g^{* *}(x, v) \geq g^{* *}(x, \alpha(x))
$$

for all $v \geq \alpha(x)$.

In fact, if $v>\alpha(x)$ is such that $g^{* *}(x, v)<g^{* *}(x, \alpha(x))$, then we can choose $w<\alpha(x)$ such that $\lambda w+(1-\lambda) v=\alpha(x)$. Since $g^{* *}(x, w)=g^{* *}(x, \alpha(x))$, we get

$$
\lambda g^{* *}(x, w)+(1-\lambda) g^{* *}(x, v)<g^{* *}(x, \alpha(x))=g^{* *}(x, \lambda w+(1-\lambda) v)
$$

in contradiction with the convexity of the function $t \rightarrow g^{* *}(x, t)$.

Now let $v, w>0$ with $v>w$. If $0<w<v<\alpha(x)$, we have

$$
g^{* *}(x, v)=g^{* *}(x, \alpha(x))=g^{* *}(x, w)
$$


if $0<w<\alpha(x)<v$, we have

$$
g^{* *}(x, v) \geq g^{* *}(x, \alpha(x))=g^{* *}(x, w)
$$

assume now that $\alpha(x)<w<v$ and $g^{* *}(x, v)<g^{* *}(x, w)$, then there exists $\lambda \in(0,1)$ such that $w=\lambda v+(1-\lambda) \alpha(x)$, so we get

$$
\begin{aligned}
\lambda g^{* *}(x, v)+(1-\lambda) g^{* *}(x, \alpha(x)) & <\lambda g^{* *}(x, w)+(1-\lambda) g^{* *}(x, w) \\
& =g^{* *}(x, w) \\
& =g^{* *}(x, \lambda v+(1-\lambda) \alpha(x)),
\end{aligned}
$$

again in contradiction with the convexity of the function $g^{* *}$.

Since $g^{* *}(x, t)$ is not decreasing, $g^{* *}(x,|A|)$ is a convex function less than $f$, so we obtain $g^{* *}(x,|A|)<f^{* *}(x, A)$.

Therefore, we have proved that $g^{* *}(x,|A|)=f^{* *}(x, A)$. We are going now to prove that $R f=f^{* *}$.

We observe that

$$
g^{* *}(x,|A|)=f^{* *}(x, A) \leq R f(x, A) .
$$

When $|A| \geq \alpha(x)$, by (2.46),

$$
g^{* *}(x,|A|)=g(x,|A|)=f(x, A) \geq R f(x, A) .
$$

So we can reduce to the case $|A|<\alpha(x)$ when $g^{* *}(x,|A|)=g^{* *}(x, \alpha(x))$.

Let $A=\left(A_{j}^{i}\right)_{1 \leq i \leq m, 1 \leq j \leq n}$, with $A_{1}^{1} \neq 0$, and define

$$
\lambda=\frac{1}{2}\left(1+\frac{\left|A_{1}^{1}\right|}{\left(\alpha(x)^{2}-|A|^{2}+\left|A_{1}^{1}\right|^{2}\right)^{1 / 2}}\right),
$$

we have $1 / 2<\lambda<1$. Let $E=\left(E_{j}^{i}\right)_{1 \leq i \leq m, 1 \leq j \leq n}$ be such that

$$
E_{1}^{1}=\frac{2 A_{1}^{1}}{1-2 \lambda}, \quad E_{j}^{i}=0, \quad i>1, j>1
$$

and let

$$
B=A-(1-\lambda) E, \quad C=A+\lambda E .
$$

Therefore, we have that

$$
A=\lambda B+(1-\lambda) C, \quad|B|=|C|=\alpha(x), \quad \operatorname{rank}(B-C) \leq 1,
$$

so by the characterization of the rank-one convex envelope,

$$
R f(x, A) \leq \lambda f(x, B)+(1-\lambda) f(x, C)=g^{* *}(x, \alpha(x))=f^{* *}(x, A) .
$$


As a final remark, we point out that condition (1.6), that is, $Q f(x, \xi)=f^{* *}(x, \xi)$, is connected with the Lavrentiev phenomenon. In an abstract framework, let $X$ and $Y$ be two topological spaces of weakly differentiable functions, with $Y \subset X$ and $Y$ dense in $X$. We say that there is the Lavrentiev phenomenon when

$$
\inf _{u \in X} \int f(x, D u) d x<\inf _{u \in Y} \int f(x, D u) d x \text {. }
$$

The following result holds.

Corollary 2.9. Under the hypotheses of Theorem 2.6, the Lavrentiev phenomenon (2.62) does not occur.

Proof. Define, as in Buttazzo and Mizel [1], the Lavrentiev gap:

$$
\mathscr{L}(u)=\mathscr{F}_{Y}(u)-\mathscr{F}_{X}(u), \quad \mathscr{L}(u)=0 \quad \text { if } \mathscr{F}_{X}(u)=+\infty,
$$

where

$$
\begin{aligned}
& \mathscr{F}_{X}(u)=\sup \{G: X \longrightarrow[0,+\infty]: G \text { l.s.c, } G \leq I \text { on } X\} \\
& \mathscr{F}_{Y}(u)=\sup \{G: X \longrightarrow[0,+\infty]: G \text { l.s.c, } G \leq I \text { on } Y\} .
\end{aligned}
$$

Since in our case $X=W^{1, p}\left(U, \mathbb{R}^{m}\right)$ and $Y=W^{1, q}\left(U, \mathbb{R}^{m}\right), U \Subset \Omega$ and

$$
\mathscr{F}_{Y}(u, U)=\mathscr{F}^{p, q}(u, U)=I^{* *}(u, U) \leq \mathscr{F}_{X}(u, U),
$$

we conclude that $\mathscr{L}(u)=0$.

Therefore, Proposition 2.8 exhibits a class of functions satisfying the condition (B1) (i.e., $f(x, \xi)=g(x,|\xi|))$ for which the Lavrentiev phenomenon does not occur.

\section{Relaxation and regularity}

In this section, we apply the relaxation equality contained in Theorem 2.6 to get the $W^{1, \infty}$ regularity for local minimizers of nonconvex functionals.

Let $f: \Omega \times \mathbb{R}^{n m} \rightarrow \mathbb{R}$ be a Caratheodory function satisfying (A1) and (A2) and the following assumptions:

(B1) there exist $R>0$ and a function $g$ such that, for almost everywhere $x \in \Omega$ and every $\xi \in \mathbb{R}^{n m} \backslash B_{R}(0)$,

$$
f(x, \xi)=g(x,|\xi|)
$$

(B2) $f$ is $p$-uniformly convex at infinity, with $p \leq q$, that is, there exist $p>1$ and $v>0$ such that, for almost everywhere $x \in \Omega$ and for every $\xi_{1}, \xi_{2} \in \mathbb{R}^{n m} \backslash B_{R}(0)$ endpoints of a segment contained in the complement of $B_{R}(0)$,

$$
\frac{1}{2}\left[f\left(x, \xi_{1}\right)+f\left(x, \xi_{2}\right)\right] \geq f\left(x, \frac{\xi_{1}+\xi_{2}}{2}\right)+\nu\left(1+\left|\xi_{1}\right|^{2}+\left|\xi_{2}\right|^{2}\right)^{(p-2) / 2}\left|\xi_{1}-\xi_{2}\right|^{2}
$$


(B3) for almost everywhere $x \in \Omega$ and every $\xi \in \mathbb{R}^{n m} \backslash B_{R}(0)$, let $D_{t}^{+} g(x,|\xi|)$ be the right-side derivative of $g$ with respect to $t$ and denote

$$
D_{\xi_{i}^{\alpha}}^{+} f(x, \xi)=D_{t}^{+} g(x,|\xi|) \frac{\xi_{i}^{\alpha}}{|\xi|} .
$$

Then for every $\xi \in \mathbb{R}^{n m} \backslash B_{R}(0)$, the vector field $x \mapsto D_{\xi}^{+} f(x, \xi)$ is weakly differentiable and

$$
\left|D_{x} D_{\xi}^{+} f(x, \xi)\right| \leq L_{1}(1+|\xi|)^{q-1}
$$

Let

$$
I(v, \Omega)=\int_{\Omega} f(x, D v) d x
$$

Cupini, et al. in [2, Theorem 1.1] proved the following regularity result for the local minimizer of $I$ when $f$ is convex with respect to $\xi$.

Theorem 3.1. Let $u$ be a local minimizer of (3.5) whose integrand $f$ is convex with respect to $\xi$ and satisfies the assumptions (A1), (B1), (B2), and (B3), $1<p \leq q<p(n+1) / n$. Then $u$ is locally Lipschitz continuous, and, for all $B_{r}\left(x_{0}\right) \Subset \Omega$,

$$
\sup _{B_{r / 4}\left(x_{0}\right)}|D u| \leq c\left[\int_{B_{r}\left(x_{0}\right)}(1+f(x, D u)) d x\right]^{\beta},
$$

where $c=c\left(n, p, q, L, L_{1}, R, v\right)$ and $\beta=\beta(n, p, q)$.

In the sequel, we need some properties of functions satisfying (A1), (A2), and the p-uniformly convexity at infinity. In [2, Lemma 2.2], it is proved that assumption (B2) implies that $f$ is $p$-coercive, that is, there exist $c_{0}, c_{1}>0$ such that

$$
c_{1}|\xi|^{p}-c_{0} \leq f(x, \xi) .
$$

By [2, Theorem 2.5(iv)], it follows that if $f$ satisfies the condition (B2), there exists $R_{0}$ depending on $v, p, q, L$ such that

$$
f^{* *}(x, \xi)=f(x, \xi),
$$

for almost everywhere $x \in \Omega$ and $\xi \in \mathbb{R}^{n m} \backslash B_{R_{0}}(0)$.

Moreover, by assumption (A2) and Remark 2.1, it is easy to check that there exists $\delta>0$ such that, for every $\xi \in B_{R_{0}}(0)$ and $x \in U$, with $U \Subset \Omega$ and $\operatorname{diam} U<\delta$, there exists $x_{0} \in U$ such that

$$
f(x, \xi) \leq \max _{\xi \in B_{R_{0}}(0)} f\left(x_{0}, \xi\right)[1+\mu(\delta)]+\mu(\delta),
$$

then, for every $\xi_{1}, \xi_{2} \in B_{R_{0}}(0)$,

$$
\left|f\left(x, \xi_{1}\right)-f\left(x, \xi_{2}\right)\right| \leq c\left[\max _{\xi \in B_{R_{0}}} f\left(x_{0}, \xi\right)+1\right]=c_{2}\left(R_{0}, U\right),
$$

where $c, c_{2}$ are positive constants. 
We now consider $\xi_{1}, \xi_{2} \in \mathbb{R}^{n m} \backslash B_{R_{0}}(0)$. By (3.8) and the growth condition, we obtain

$$
\left|f\left(x, \xi_{1}\right)-f\left(x, \xi_{2}\right)\right| \leq c_{3}\left[1+\left|\xi_{1}\right|+\left|\xi_{2}\right|\right]^{q-1}\left|\xi_{1}-\xi_{2}\right|
$$

with $c_{3}=c_{3}(L, q)$.

Therefore, it is easy to check that there exist $c_{2}=c_{2}\left(R_{0}, U\right)$ and $c_{3}(L, q)$ such that

$$
\left|f\left(x, \xi_{1}\right)-f\left(x, \xi_{2}\right)\right| \leq c_{2}+c_{3}\left[1+\left|\xi_{1}\right|+\left|\xi_{2}\right|\right]^{q-1}\left|\xi_{1}-\xi_{2}\right|
$$

Finally, for every $x \in U$, where $U \Subset \Omega$, and for every $\xi \in \mathbb{R}^{n m}$, (3.8) implies that

$$
f(x, t \xi) \leq c_{4}(1+f(x, \xi))
$$

for all $t \in[0,1]$, where $c_{4}$ depends on $v$ and $R_{0}$.

Our main result is the following regularity theorem.

Theorem 3.2. Let $f$ satisfy (A1), (A2), (B1), (B2), and (B3), with $1<p<q<p(n+1) / n$ and $Q f(x, \xi)=f^{* *}(x, \xi)$. Let $u$ be a local minimizer of the functional I in (3.5), then $u \in$ $W_{\text {loc }}^{1, \infty}\left(\Omega, \mathbb{R}^{m}\right)$ and estimate (3.6) holds.

Proof. We suppose that for every $U \Subset \Omega, u$ is a local minimizer and then $u$ is a solution of the following boundary value problem:

$$
I(u)=\inf \left\{I(v, U), v \in W_{0}^{1, p}\left(\Omega, \mathbb{R}^{m}\right)+u\right\} .
$$

Consider the relaxed problem of (3.14):

$$
I^{* *}(u)=\inf \left\{I^{* *}(v, U), v \in W_{0}^{1, p}\left(\Omega, \mathbb{R}^{m}\right)+u\right\},
$$

where, as usual,

$$
I^{* *}(v, U)=\int_{U} f^{* *}(x, D v) d x
$$

By the convexity of $I^{* *}$, the problem (3.15) has at least a solution $\bar{u} \in W_{0}^{1, p}\left(\Omega, \mathbb{R}^{m}\right)+u$. Since $\bar{u}$ is also a local minimizer of $I^{* *}$ and $f^{* *}$ satisfies (A1), (B1), (B2), and (B3) in $\Omega \times \mathbb{R}^{n m} \backslash B_{R_{0}}(0)$, then we can apply Theorem 3.1 and so we obtain $\bar{u} \in W_{\text {loc }}^{1, \infty}\left(U, \mathbb{R}^{m}\right)$.

Observe that $f(x, D \bar{u}) \in L^{1}(U)$. In fact, we can suppose, without loss of generality, that $\operatorname{diam} U<\delta$; then, for $\xi \in B_{R_{0}}(0)$, by (3.9),

$$
f(x, \xi) \leq c_{2}\left(R_{0}, U\right)
$$

and then we get

$$
\begin{aligned}
\int_{U} f(x, D \bar{u}) d x & =\int_{U \cap\left\{x:|D \bar{u}| \leq R_{0}\right\}} f(x, D \bar{u}) d x+\int_{U \cap\left\{x:|D \bar{u}|>R_{0}\right\}} f^{* *}(x, D \bar{u}) d x \\
& \leq c_{2}\left(R_{0}, U\right)|U|+\int_{U} f^{* *}(x, D \bar{u}) d x<\infty .
\end{aligned}
$$


Let $\left(u_{k}\right) \subset W^{1, q}\left(U, \mathbb{R}^{m}\right)$, with $u_{k} \rightarrow \bar{u} w-W^{1, p}\left(U, \mathbb{R}^{m}\right)$; for $\varepsilon>0$, let $\Sigma \Subset U, \Sigma$ open, such that

$$
I(\bar{u}, U \backslash \Sigma) \leq \varepsilon
$$

and let $M>0$ be such that

$$
\|D \bar{u}\|_{\infty, \Sigma} \leq M
$$

Moreover, let $\Sigma_{0} \Subset \Sigma$ and let $\nu \in \mathbb{N}$. For $i=1, \ldots, \nu$, we define

$$
\Sigma_{i}=\left\{x \in \Sigma: \operatorname{dist}\left(x, \Sigma_{0}\right)<\frac{i}{\nu} R\right\}
$$

where $R=\operatorname{dist}\left(\Sigma_{0}, \partial \Sigma\right)$. For $i=1, \ldots, \nu$, consider the scalar functions $\varphi_{i} \in C_{0}^{1}\left(\Sigma_{i}\right)$ such that

$$
0 \leq \varphi_{i} \leq 1, \quad \varphi_{i}(x)=\left\{\begin{array}{ll}
1 & \text { in } \Sigma_{i-1}, \\
0 & \text { in } \Sigma \backslash \Sigma_{i},
\end{array} \quad\left|D \varphi_{i}\right| \leq \frac{v+1}{R} .\right.
$$

For all $k \in \mathbb{N}$, define

$$
v_{k i}=\left(1-\varphi_{i}\right) \bar{u}+\varphi_{i} u_{k}
$$

We have $v_{k i}=u_{k}$ in $\Sigma_{i-1}$ and $v_{k i}=\bar{u}$ in $\Sigma \backslash \Sigma_{i-1}$. Define $\tilde{v}_{k i}=v_{k i}$ in $\Sigma$ and $\tilde{v}_{k i}=\bar{u}$ in $U \backslash \Sigma$.

Consider

$$
\begin{aligned}
\int_{U} f\left(x, D \tilde{v}_{k i}\right) d x= & \int_{U \backslash \Sigma} f(x, D \bar{u}) d x+\int_{\Sigma \backslash \Sigma_{i}} f(x, D \bar{u}) d x \\
& +\int_{\Sigma_{i-1}} f\left(x, D u_{k}\right) d x+\int_{\Sigma_{i} \backslash \Sigma_{i-1}} f\left(x, D v_{k i}\right) d x,
\end{aligned}
$$

then we obtain

$$
\begin{aligned}
\int_{U} f\left(x, D \tilde{v}_{k i}\right) d x \leq & \varepsilon+\int_{\Sigma \backslash \Sigma_{i}} f(x, D \bar{u}) d x+\int_{\Sigma_{i-1}} f\left(x, D u_{k}\right) d x \\
& +\int_{\Sigma_{i} \backslash \Sigma_{i-1}}\left[f\left(x, D v_{k i}\right)-f\left(x, \varphi_{i} D u_{k}\right)\right] d x \\
& +\int_{\Sigma_{i} \backslash \Sigma_{i-1}} f\left(x, \varphi_{i} D u_{k}\right) d x .
\end{aligned}
$$

Inequality (3.12) gives

$$
\begin{aligned}
& J_{k}^{i}= \int_{\Sigma_{i} \backslash \Sigma_{i-1}}\left[f\left(x, D v_{k i}\right)-f\left(x, \varphi_{i} D u_{k}\right)\right] d x \\
& \leq c_{2}\left|\Sigma_{i} \backslash \Sigma_{i-1}\right|+c_{3} \int_{\Sigma_{i} \backslash \Sigma i-1}\left[1+\left|D v_{k i}\right|+\left|\varphi_{i} D u_{k}\right|\right]^{q-1}\left|D v_{k i}-\varphi_{i} D u_{k}\right| d x \\
& \leq c_{2}\left|\Sigma_{i} \backslash \Sigma_{i-1}\right|+c_{3} \int_{\Sigma_{i} \backslash \Sigma i-1}\left[1+|D \bar{u}|+2\left|D u_{k}\right|+\left|D \varphi_{i}\right|\left|u_{k}-\bar{u}\right|\right]^{q-1} \\
& \times\left[\left(1-\varphi_{i}\right)|D \bar{u}|+\left|D \varphi_{i}\right|\left|u_{k}-\bar{u}\right|\right] d x
\end{aligned}
$$


By applying the Holder inequality with the exponents $p /(q-1)$ and $p /(p-q+1)$, we obtain

$$
\begin{aligned}
\sum_{i=1}^{\nu} J_{k}^{i} \leq & c_{2}\left|\Sigma \backslash \Sigma_{0}\right|+c_{3}|| 1+|D \bar{u}|+2\left|D u_{k}\right|+\frac{\nu+1}{R}\left|u_{k}-\bar{u}\right| \|_{L^{p}}^{q-1} \\
& \times\left(M\left|\Sigma \backslash \Sigma_{0}\right|^{(p-q+1) / p}+\frac{\nu+1}{R}\left\|u_{k}-\bar{u}\right\|_{L^{p /(p-q+1)}\left(\Sigma_{\nu}\right)}\right) .
\end{aligned}
$$

Taking in account that the sequence $\left(D u_{k}\right)$ has a bounded norm in $L^{p}\left(\Omega, \mathbb{R}^{n m}\right)$ and that, since the assumption $q<p(n+1) / n$ implies $p /(p-q+1)<p^{*}$ and $\left(u_{k}\right)$ converges $s-L^{p /(p-q+1)}\left(\Omega, \mathbb{R}^{m}\right)$ to $\bar{u}$, we can conclude that there exists a constant $c_{5}$ independent of $k$ and $v$ such that

$$
\limsup _{k \rightarrow \infty} \sum_{i=1}^{v} J_{k}^{i} \leq c_{5} .
$$

Observe that, since $v_{k i} \in W_{0}^{1, p}\left(U, \mathbb{R}^{m}\right)+u$ and $\bar{u}=u$ on $\partial U$, by the minimality of $u$ for problem (3.14), we get $I(u, U) \leq I\left(\tilde{v}_{k i}, U\right)$, for all $i$ for all $k$.

Therefore, summing up with respect to $i=1, \ldots, v$ in $(3.25)$, we get

$$
\begin{aligned}
\nu \int_{U} f(x, D u) d x \leq & \nu \mathcal{E}+\sum_{i=1}^{v} \int_{\Sigma \backslash \Sigma_{i}} f(x, D \bar{u}) d x+v \int_{\Sigma} f\left(x, D u_{k}\right) d x \\
& +c_{5}+\sum_{i=1}^{v} \int_{\Sigma_{i} \backslash \Sigma_{i-1}} f\left(x, \varphi_{i} D u_{k}\right) d x
\end{aligned}
$$

Moreover, since (3.13) implies that

$$
f\left(x, \varphi_{i} D u_{k}\right) \leq c_{4}\left[1+f\left(x, D u_{k}\right)\right]
$$

we obtain

$$
\int_{U} f(x, D u) d x \leq \varepsilon+\frac{v+c_{6}}{\nu} \int_{U} f\left(x, D u_{k}\right) d x+\frac{c_{5}}{v}+\frac{1}{\nu} \int_{\Sigma \mid \Sigma_{0}} f(x, D \bar{u}) d x .
$$

As $k$ goes to infinity, we get

$$
\int_{U} f(x, D u) d x \leq \varepsilon+\frac{v+c_{6}}{v} \liminf _{k \rightarrow \infty} \int_{U} f\left(x, D u_{k}\right) d x+\frac{c_{5}}{v}+\frac{1}{v} \int_{\Sigma \backslash \Sigma_{0}} f(x, D \bar{u}) d x .
$$

Then passing to the limit $v \rightarrow \infty$,

$$
\int_{U} f(x, D u) d x \leq \varepsilon+\liminf _{k \rightarrow \infty} \int_{U} f\left(x, D u_{k}\right) d x .
$$

Taking the infimum over the sequences $\left(u_{k}\right)$,

$$
\int_{U} f(x, D u) d x \leq \mathscr{F}^{p, q}(\bar{u}, U) .
$$


Since $Q f(x, \xi)=f^{* *}(x, \xi)$, by Theorem 2.6, we have

$$
\int_{U} f(x, D u) d x \leq \int_{U} f^{* *}(x, D \bar{u}) d x .
$$

On the other hand, $\bar{u}$ is a solution of (3.15), then

$$
\int_{U} f(x, D u) d x=\int_{U} f^{* *}(x, D \bar{u}) d x=\int_{U} f^{* *}(x, D u) d x,
$$

which implies that $u$ is also a solution of (3.15). Theorem 2.2 ensures that $u \in W_{\text {loc }}^{1, \infty}(U$, $\left.\mathbb{R}^{m}\right)$; for the arbitrariness of $U$, we get $u \in W_{\text {loc }}^{1, \infty}\left(\Omega, \mathbb{R}^{m}\right)$. Estimate (3.6) follows easily.

Remark 3.3. We observe that the arguments of the proof of Theorem 3.2 show that, for all $\bar{u} \in W_{\text {loc }}^{1, \infty}\left(\Omega, \mathbb{R}^{m}\right)$ and for all $U \Subset \Omega$, we have

$$
\mathscr{F}_{0}(\bar{u}, U)=\mathscr{F}^{p, q}(\bar{u}, U)=\int_{U} f^{* *}(x, D \bar{u}) d x,
$$

where

$$
\begin{array}{r}
\mathscr{F}_{0}(\bar{u}, U)=\inf _{u_{k}}\left\{\liminf _{k \rightarrow \infty} \int_{\Omega} f\left(x, D u_{k}\right) d x,\left(u_{k}\right) \in W_{0}^{1, p}\left(\Omega, \mathbb{R}^{m}\right)+\bar{u},\right. \\
\left.u_{k} \rightarrow \bar{u} \text { in } W^{1, p}\left(\Omega, \mathbb{R}^{m}\right), f\left(x, D u_{k}\right) \in L^{1}(\Omega)\right\} .
\end{array}
$$

Indeed, back to the proof of Theorem 3.2, the sequence $\tilde{v}_{k i} \in W_{0}^{1, p}\left(U, \mathbb{R}^{m}\right)+\bar{u}$ is such that $f\left(x, D \tilde{v}_{k i}\right) \in L^{1}(U)$ and $\tilde{v}_{k i} \rightarrow \bar{u}$ in $W^{1, p}\left(\Omega, \mathbb{R}^{m}\right)$. Therefore, taking the lower bound in (3.25) and summing up with respect to $i=1, \ldots, \nu$ as $k \rightarrow \infty$, we have

$$
\mathscr{F}_{0}(\bar{u}, U) \leq \varepsilon+\frac{v+c_{6}}{v} \liminf _{k \rightarrow \infty} \int_{U} f\left(x, D u_{k}\right) d x+\frac{c_{5}}{v}+\frac{1}{v} \int_{\Sigma \backslash \Sigma_{0}} f(x, D \bar{u}) d x .
$$

As $v \rightarrow \infty$, we get

$$
\mathscr{F}_{0}(\bar{u}, U) \leq \mathscr{F}^{p}, q(\bar{u}, U) \leq \mathscr{F}_{0}(\bar{u}, U)
$$

\section{References}

[1] G. Buttazzo and V. J. Mizel, Interpretation of the Lavrentiev phenomenon by relaxation, J. Funct. Anal. 110 (1992), no. 2, 434-460.

[2] G. Cupini, M. Guidorzi, and E. Mascolo, Regularity of minimizers of vectorial integrals with $p-q$ growth, Nonlinear Anal. 54 (2003), no. 4, 591-616.

[3] G. Cupini and A. P. Migliorini, Hölder continuity for local minimizers of a nonconvex variational problem, J. Convex Anal. 10 (2003), no. 2, 389-408.

[4] B. Dacorogna, Direct Methods in the Calculus of Variations, Applied Mathematical Sciences, vol. 78, Springer-Verlag, Berlin, 1989.

[5] E. De Giorgi, Sulla convergenza di alcune successioni d'integrali del tipo dell'area, Rend. Mat. (6) 8 (1975), 277-294 (Italian). 


\section{Regularity of minimizers for nonconvex integrals}

[6] L. Esposito, F. Leonetti, and G. Mingione, Regularity results for minimizers of irregular integrals with $(p, q)$ growth, Forum Math. 14 (2002), no. 2, 245-272.

[7] I. Fonseca, N. Fusco, and P. Marcellini, An existence result for a nonconvex variational problem via regularity, ESAIM Control Optim. Calc. Var. 7 (2002), 69-95.

[8] I. Fonseca and J. Malý, Relaxation of multiple integrals below the growth exponent, Ann. Inst. H. Poincaré Anal. Non Linéaire 14 (1997), no. 3, 309-338.

[9] N. Fusco, On the convergence of integral functionals depending on vector-valued functions, Ricerche Mat. 32 (1983), no. 2, 321-339.

[10] E. Giusti, Metodi Diretti nel Calcolo delle Variazioni [Direct Methods in the Calculus of Variations], Unione Matematica Italiana, Bologna, 1994 (Italian).

[11] P. Marcellini, Approximation of quasiconvex functions, and lower semicontinuity of multiple integrals, Manuscripta Math. 51 (1985), no. 1-3, 1-28.

[12] - Regularity of minimizers of integrals of the calculus of variations with nonstandard growth conditions, Arch. Ration. Mech. Anal. 105 (1989), no. 3, 267-284.

[13] E. Mascolo and A. P. Migliorini, Everywhere regularity for vectorial functionals with general growth, ESAIM Control Optim. Calc. Var. 9 (2003), 399-418.

[14] E. Mascolo and R. Schianchi, Existence theorems in the calculus of variations, J. Differential Equations 67 (1987), no. 2, 185-198.

Irene Benedetti: Dipartimento di Matematica "Ulisse Dini," Università di Firenze, Viale Morgagni 67/A, 50134 Firenze, Italy

E-mail address: benedetti@math.unifi.it

Elvira Mascolo: Dipartimento di Matematica “Ulisse Dini,” Università di Firenze, Viale Morgagni 67/A, 50134 Firenze, Italy

E-mail address: mascolo@math.unifi.it 


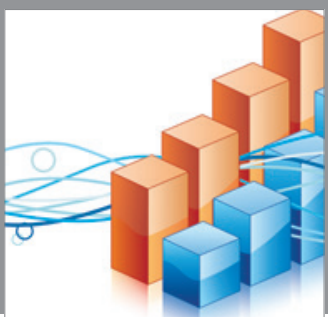

Advances in

Operations Research

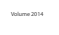

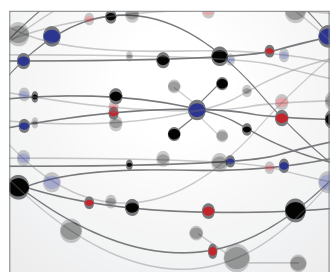

\section{The Scientific} World Journal
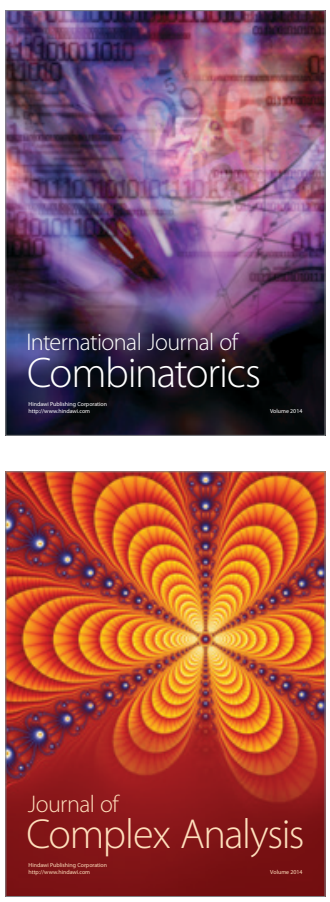

International Journal of

Mathematics and

Mathematical

Sciences
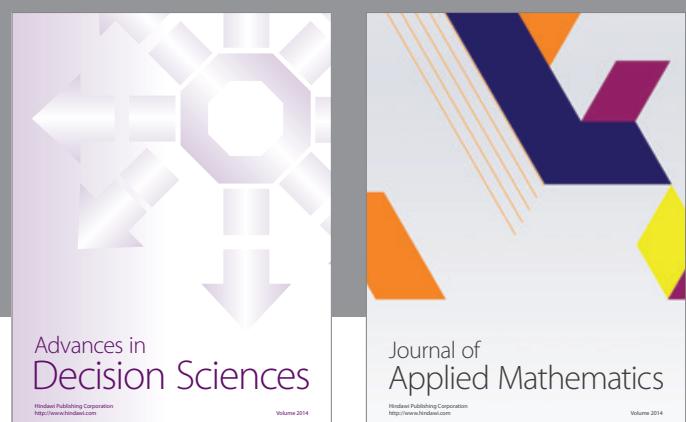

Journal of

Applied Mathematics
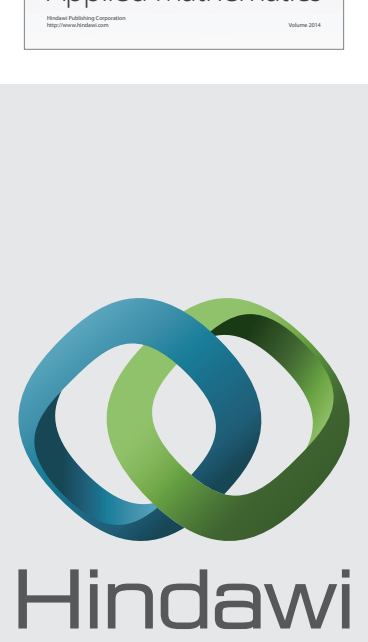

Submit your manuscripts at http://www.hindawi.com
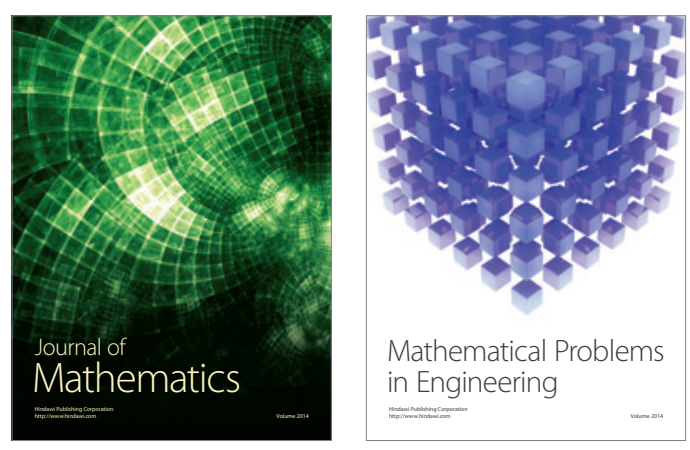

Mathematical Problems in Engineering
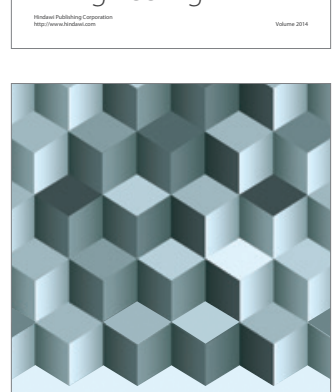

Journal of

Function Spaces
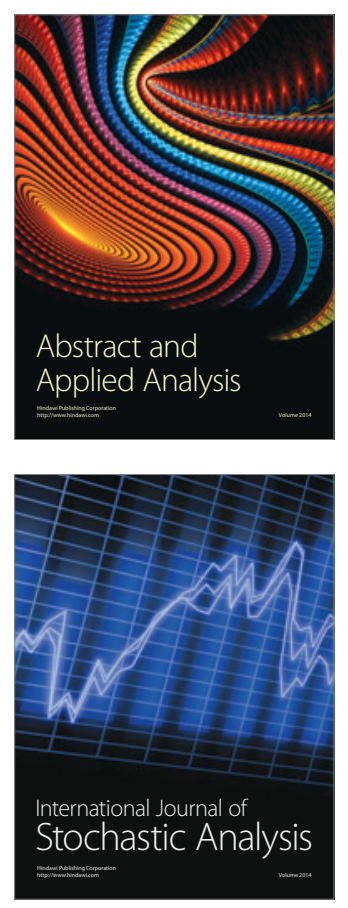

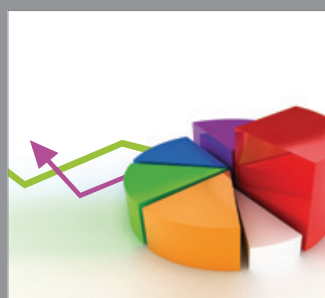

ournal of

Probability and Statistics

Promensencen
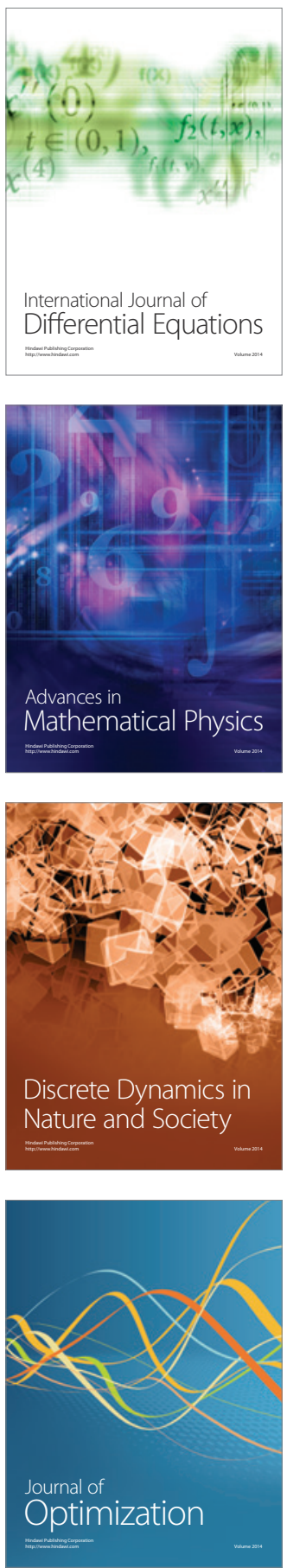